\title{
Introducing DeepBalance: Random Deep Belief Network Ensembles to Address Class Imbalance
}

\author{
Peter Xenopoulos ${ }^{1}$ \\ peter.xenopoulos@pomona.edu
}

\begin{abstract}
Class imbalance problems manifest in domains such as financial fraud detection or network intrusion analysis, where the prevalence of one class is much higher than another. Typically, practitioners are more interested in predicting the minority class than the majority class, as the minority class may carry a higher misclassification cost. However, classifier performance deteriorates in the face of class imbalance as oftentimes classifiers may predict every point as the majority class. Methods for dealing with class imbalance include costsensitive learning or resampling techniques. In this paper, we introduce DeepBalance, an ensemble of deep belief networks trained with balanced bootstraps and random feature selection. We demonstrate that our proposed method outperforms baseline resampling methods such as SMOTE and under- and oversampling in metrics such as AUC and sensitivity when applied to highly imbalanced financial transaction data sets. Additionally, we explore performance and training time implications of various model parameters. Furthermore, we show that our model is easily parallelizable, which can reduce training times. Finally, we present an implementation of DeepBalance in $R$.
\end{abstract}

\section{INTRODUCTION}

When solving practical classification problems, a practitioner may be faced with class imbalance, meaning that one class has a significantly higher prevalence than the others (also called the majority class). Examples of imbalanced classification problems in the literature include [1], [2], [3], [4]. Class imbalance problems may be exacerbated in the future as we discover new methods to collect rare data and rate of data collection increases. In many class imbalance problems, the minority class is not only the interest, but also carries the higher misclassification cost, which complicates learning [5].

Machine learning classifiers try to find an optimal decision boundary that fits training data. As classifiers generally seek to find the simplest rule that partitions the training data, the simplest rule in imbalanced settings is often always predicting the majority class [6]. Results can be deceptive for such classifiers, as they may achieve high accuracy. For example, in a problem where a minority class occurs $0.1 \%$ of the time, an uninformed classifier can achieve 99.9\% accuracy by simply always predicting observations as the majority. Thus, the naturally occurring target class distribution is not optimal for learning in highly imbalanced scenarios [7], [8], [9], [10].

Two common methods exist for dealing with class imbalance: resampling methods that balance the data before model training or cost-sensitive learning methods that adjust

\footnotetext{
${ }^{1} \mathrm{P}$. Xenopoulos is a student in the mathematics and economics departments at Pomona College in Claremont, CA 91711
}

the relative costs of the errors during model training. While cost-sensitive methods have been shown to alleviate learning difficulties for neural networks in the wake of class imbalance, we investigate the use of resampling methods, as costsensitive methods may be hard to implement for practitioners or error costs may be unknown [11]. Examples of resampling methods include under- and over-sampling, SMOTE and ROSE [12], [13], [14]. While under- and over-sampling are some of the simplest methods to implement, they also carry some drawbacks. Under-sampling may discard useful information in an effort to create a balanced data set, and oversampling may increase the likelihood of model overfitting [15], [16], [17]. SMOTE builds upon these two methods by up-sampling the minority class and down-sampling the majority class. Further research related to SMOTE, such as SMOTEboost and Borderline-SMOTE, have shown its ability to increase the performance of underlying classifiers when applied to imbalanced class problems [18], [19]. These sampling techniques have also been shown to increase the performance of support vector machines across a variety of problems by reducing bias towards the majority class [20], [21]. However, beyond SVMs and tree-based methods, the effects of class imbalance are still relatively unexplored [22], [23].

In this paper, we focus on prediction improvements through resampling methods by applying ensemble methodology similar to balanced random forests or EasyEnsemble [24], [25]. Part of the power of these methods revolve around ensemble learning [26]. Specifically, the constituent base classifier in our ensemble is the deep belief network (DBN). Previous studies have shown that simple approaches which combine random undersampling with bagging or boosting ensembles are favorable in the wake of class imbalance [27]. Furthermore, these studies noted a particular synergy between random undersampling and bagging. Here, we present DeepBalance, an ensemble of deep belief networks, trained with random feature selection on balanced boostraps. Additionally, we provide an implementation of DeepBalance in R. Finally, we show that DeepBalance is easily parallelizable, making training times more palatable to practitioners.

The rest of the paper is as follows. In section II, we present related mathematical background and our algorithm, DeepBalance. In section III we outline our experimental methods. In section IV we present our results. In section $\mathrm{V}$ we present a discussion of our results and in section VI we conclude the paper. 


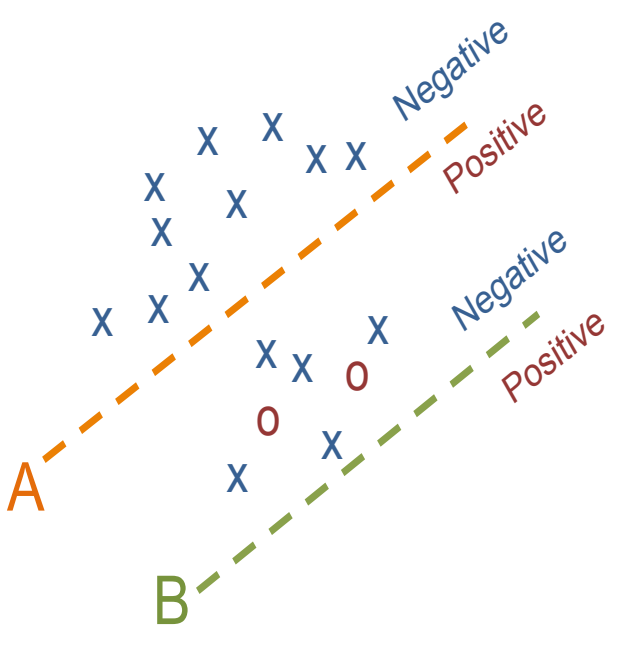

Fig. 1. An illustrated class imbalance problem.

\section{Methods}

\section{A. Class Imbalance}

Suppose we have a classifier, $C$, which separates a space into two. Let us also assume that $C$ is designed to minimize error rate, and thus maximize accuracy, defined as

$$
\frac{\text { Misclassifications }}{\text { Observations }}
$$

In an imbalanced case, the loss may be dominated by misclassification of the majority class.

In Figure 1, we have two classifiers, $A$ and $B$. We see that the misclassification error of $\mathrm{A}$ is $5 / 17$, as we misclassify the five blue x's on the "Positive" partition of the classifier. We also see that the misclassification error of $\mathrm{B}$ is $2 / 17$. Thus, we would choose classifier B to maximize our accuracy, even though we misclassify all of the positive cases (red points).

As mentioned previously, there are two approaches to deal with this imbalance: cost-sensitive learning and resampling methods. Although seemingly different, these two approaches are quite similar in that both seek to adjust for the different costs associated with errors. Cost-sensitive learning changes the loss function to account for the usually higher cost associated with misclassifying a minority class as the majority. One such modification could include adding a penalty for each positive case missed.

However, dealing with loss functions can be hard for practitioners, as they may lack the ability to directly modify or determine costs. Resampling indirectly adjusts for costs by evening out the class distributions and requires no modification of the loss function. For example, oversampling of the minority increases the cost of misclassifying a minority observation by sampling the same points multiple times. If you oversample an original observation, you now have two of the same observation in your training set. Thus, if your classifier misclassifies that point, it misclassifies it twice.

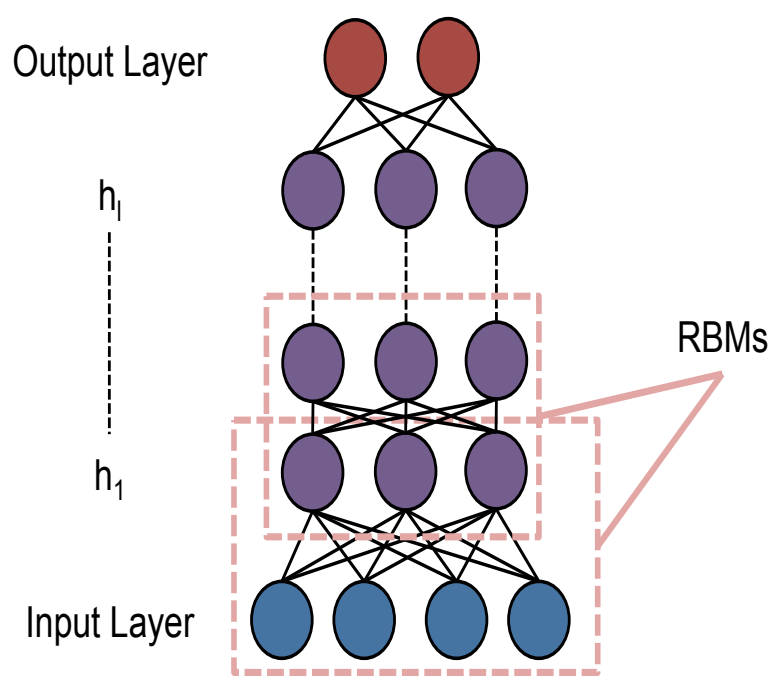

Fig. 2. An example of a basic deep belief network for binary classification with $k$ hidden layers

\section{B. Deep Belief Networks}

The base learner of our proposed ensemble is the deep belief network (DBN) architecture described in [28], [29]. A DBN is composed of multiple restricted Boltzmann machines (RBMs) shown in Figure 2. These RBMs are stacked on top of each other, taking their inputs from the hidden layer of the previous RBM. We use DBNs as our base learners due to their ability to model complex interactions and strong performance at classification tasks [30], [31], [32]. Additionally, DBN ensembles have been employed to forecast time series, diagnose failures and to assess credit risk [33], [34], [35].

\section{DeepBalance}

We present DeepBalance in Algorithm 1. DeepBalance trains DBNs on balanced bootstraps of data, meaning the bootstrapped data is sampled such that it has equal target class prevalence. The idea of a balanced bootstrap stems from the possibility of bootstrapping samples with no minority cases, which may be especially prevalent in situations with a large class imbalance.

Each DBN is then added to our ensemble. TrainDBN represents the function we use to train a DBN and takes parameters $\varphi, \theta$, mtry and max.it. $\varphi$ and $\theta$ represent the minority-class and majority-class data, respectively. Each model is trained with at most mtry number of randomly sampled (with replacement) features from the feature space, and the sampled features are denoted as $F_{S}$. max.it specifies the maximum number of fine-tuning epochs in training a DBN. Each DBN is fine-tuned with backpropagation.

By introducing randomness in the feature selection process, as well as the sampling process, we create a more diverse ensemble, and we can help the ensemble generalize. If one only introduced randomness via the balanced bootstrap process, and the train majority data was very homogenous, the trained classifier may not generalize well to new data. 


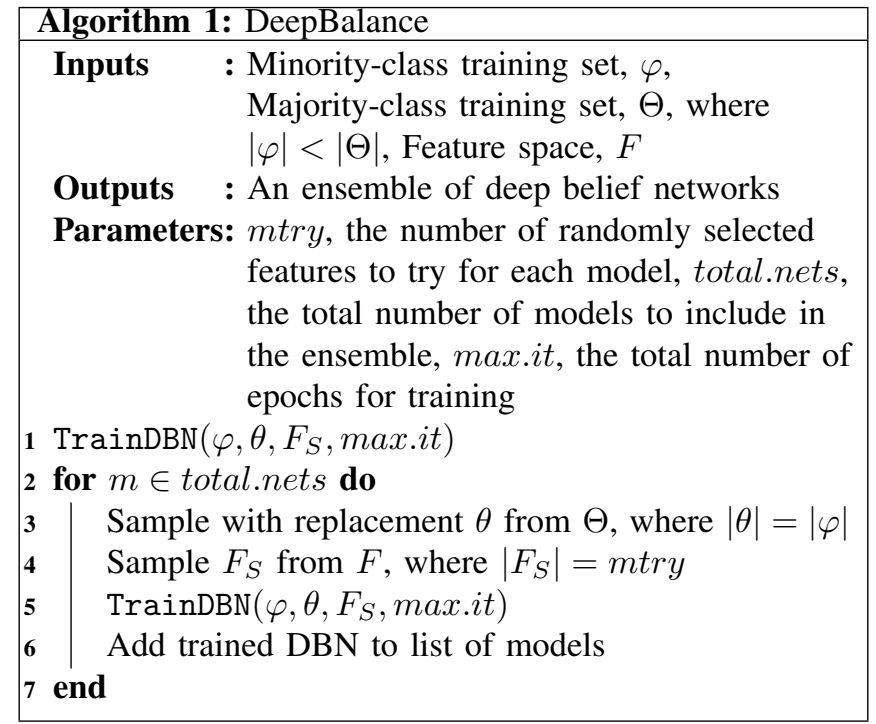

\section{METHODS}

\section{A. Data}

To benchmark DeepBalance we use financial transaction data, which is typically imbalanced. While most transactions are not fraudulent, there exist a small percentage that are fraudulent. These few fraudulent transactions usually incur costly monetary repercussions. Thus, it is important for financial transactions to be quickly and accurately classified. Our selected data necessitate the use of resampling methods as conventional train/test splits may not capture enough minority cases to train any effective classifier.

Our first data set contains hundreds of thousands of European credit card transactions made over two days in September 2013 [36]. It contains 29 features: $V_{1}, V_{2}, \ldots, V_{29}$, the principal components of a prior PCA transformation, and Amount, the dollar amount of the transaction. We drop the Time variable from the original data set as it represents time since last transaction and mostly acts as an index for the data. In total we have $n=284,807$ observations, with 492 positive fraud cases, for a fraud incidence of $0.173 \%$.

We also chose to benchmark DeepBalance on a simulated financial transaction data from the PaySim simulator [37]. PaySim is an agent-based simulation which closely mirrors financial transaction data. From this simulated data, we have $n=6,362,620$ simulated financial transactions where only 8,213 are fraudulent, for a fraud incidence of $0.129 \%$. We use the five variables described in Table I to classify the transactions.

\section{B. Sampling Methods}

We compare various sampling methods against DeepBalance, which we describe in Table II. We institute a $70 \% / 30 \%$ train/test split on each data set. Specifically, we use $70 \%$ of our positive and $70 \%$ of our negative cases, respectively, to create a training data set. The remaining $30 \%$ of each case is combined to create a testing data set. Our DeepBalance method is parameterized with total.nets $=25$
TABLE I

PAYSIM FEATURES

\begin{tabular}{ll}
\hline Features & Description \\
\hline type & Type of transaction, such as CASH-IN, CASH-OUT, \\
amount & DEBIT, PAYMENT and TRANSFER \\
oldbalanceOrig & Initial balance before the transaction from origin \\
newbalanceOrig & New balance after the transaction from origin \\
oldbalanceDest & Initial balance recipient before the transaction. \\
newbalanceDest & New balance recipient after the transaction. \\
\hline
\end{tabular}

TABLE II

RESAMPLING METHODS

Method

Over-sampling

Under-sampling

SMOTE

No resampling

All Features Ensemble

Description

Uses the resampling methods described in methods

Over-samples $x$ positive cases to create a balanced sample with $x$ positive and $x$ randomly sampled with replacement negative cases, where $x=1000$ for the credit card fraud data and $x=12,000$ for the PaySim data

Under-samples $|\phi|$ majority cases and combines with all of the minority cases

Uses the methodology in,[13] and the implementation in [38]

Uses no resampling method, uses the training data containing $70 \%$ of each class

Similar to DeepBalance, except without random feature selection and $\max . i t=50$. For the European credit card fraud data, we set $m t r y=5$, and for the PaySim data, we set $m t r y=3$. For under-sampling, over-sampling and SMOTE, we trained a DBN using all available features and set $\max . i t=100$.

\section{Performance Metrics}

For imbalanced class problems, accuracy is an inappropriate tool to gauge model performance. A classifier which always predicts the majority class can perform extremely well on an accuracy basis, if the imbalance is high. Common metrics for assessing classifier performance in binary classification problems include the true negative rate $\left(A c c^{-}\right)$and the true positive rate $\left(A c c^{+}\right)$, shown below:

$$
\begin{aligned}
& A c c^{-}=\frac{T N}{T N+F P} \\
& A c c^{+}=\frac{T P}{T P+F N}
\end{aligned}
$$

The true positive rate is also often referred to as sensitivity. The true negative rate is often referred to as specificity. Additionally, weighted accuracy adds another dimension to performance evaluation, where weighted accuracy is defined as

$$
\beta \times A c c^{-}+(1-\beta) \times A c c^{+}
$$


In this study, we set $\beta$ equal to 0.5 . When $\beta=0.5$, weighted accuracy is sometimes called balanced accuracy.

Additionally we look at the area under the curve (AUC), which we derive from the receiver operating characteristic curve. The receiver operating characteristic curve describes the ability of a classifier to identify both positive and negative classes as the decision threshold changes [39].

\section{D. $R$ Implementation}

We offer an implementation of the aforementioned DeepBalance in $\mathbf{R} 1$. The implementation of deep belief networks is provided by [40]. The main functionality of our $\mathbf{R}$ implementation is through the RNNE () function, which takes arguments formula, train, mtry, total.nets, max.it. The formula parameter contains an $\mathrm{R}$ formula of a target variable and its predictors, and is typically structured as Outcome $\sim V_{1}+V_{2}+\ldots+V_{n}$ where $V_{n}$ represents the $n^{\text {th }}$ feature. The train parameter represents a data set that contains both Outcome and $V_{1}, \ldots, V_{n}$. The mtry, total.nets and max.it parameters are the same as described in section II. Using the features passed through formula, the data is separated into data frames containing the majority and minority data (denoted as $\Theta$ and $\varphi$ ). An empty list is then created to contain all of the trained models.

In the model training process, we start by sampling with replacement the number of minority cases from $\Theta$, which we call $\theta$, where $|\theta|=|\varphi|$. We then combine $\theta$ and $\varphi$ to create our training set. Then, we sample mtry variables, with replacement, from our predictors. Finally, we train a deep belief network given our combined data, our randomly sampled features and max.it. This network is then added to the list of DBNs we initialized earlier. This process is repeated until the length of the list of DBNs is equal to total.nets. Thus, our list of DBNs is our ensemble.

\section{Results}

We present a comparison of DeepBalance versus standard resampling methods for dealing with class imbalance in both Table III and Table IV. We can observe the smoothed ROC curves for both the European credit card fraud and PaySim data in Figure 3. To calculate $\mathrm{Acc}^{+}, \mathrm{Acc}^{-}$and Balanced Accuracy, we assume a decision threshold of 0.5 for both data sets. Bold indicates the best model by respective performance metric. We do not include DBN + No resampling output for PaySim due to restrictive training times (over 4 million observations). We also do not include DBN + All Features for PaySim as its performance converges to the DeepBalance solution.

We see that DeepBalance outperforms all candidate methods on both data sets. It is important to note the especially strong performance of DeepBalance when applied to the European credit card fraud data set, which may be aided by the features being uncorrelated by virtue of the principal components.

\footnotetext{
${ }^{1}$ Our implementation of DeepBalance is currently available in the following GitHub repository: https://github.com/peterxeno/ DeepBalance
}

TABLE III

MODEL COMPARISONS (EUROPEAN CREDIT CARD FRAUD)

\begin{tabular}{ccccc}
\hline Method & $A c c^{+}$ & $A c c^{-}$ & Balanced Accuracy & AUC \\
\hline DeepBalance & $\mathbf{0 . 8 1 7 6}$ & $\mathbf{0 . 9 9 4 6}$ & $\mathbf{0 . 9 0 6 1}$ & $\mathbf{0 . 9 7 7 6}$ \\
SMOTE & 0.8176 & 0.8870 & 0.8523 & 0.9158 \\
Undersampling & 0.5338 & 0.9752 & 0.7545 & 0.8248 \\
Oversampling & 0.7500 & 0.9728 & 0.8614 & 0.8672 \\
No Resampling & 0.7027 & 0.9707 & 0.8367 & 0.8526 \\
\hline
\end{tabular}

TABLE IV

MODEL COMPARISONS (PAYSIM)

\begin{tabular}{ccccc}
\hline Method & $A c c^{+}$ & $A c c^{-}$ & Balanced Accuracy & AUC \\
\hline DeepBalance & 0.8766 & 0.9044 & $\mathbf{0 . 8 9 0 5}$ & $\mathbf{0 . 9 6 1 4}$ \\
SMOTE & 0.7618 & $\mathbf{0 . 9 4 0 2}$ & 0.7311 & 0.9175 \\
Undersampling & 0.8068 & 0.7001 & 0.7534 & 0.7590 \\
Oversampling & $\mathbf{0 . 9 1 3 1}$ & 0.8169 & 0.8650 & 0.8513 \\
\hline
\end{tabular}

DeepBalance's performance with the PaySim data illustrates an example of evaluating models across many metrics. While DeepBalance doesn't attain the highest $\mathrm{Acc}^{+}$or $\mathrm{Acc}^{-}$, we see that it scores the highest in balanced accuracy and AUC, indicating that DeepBalance achieves the best overall performance. Such conclusions would be lost if we only evaluated DeepBalance on an $A c c^{+}$or $A c c^{-}$basis.

\section{DISCUSSION}

While we see that DeepBalance outperforms conventional methods to deal with class imbalance, we are still left uncertain about the properties of its parameters, mtry, total.nets and max.it. Each of these parameters has implications on model performance and training times. Using the European credit card data, we show how DeepBalance scales in both performance and training time as we change parameters.

We see the effect of increasing mtry on average model performance (measured through AUC) and average model training time in Figure 4. For this experiment, we trained DeepBalance models with max $. i t=50$ and total.nets $=5$ across mtry $=1, \ldots, 29$ and averaged the model performance at each mtry. We used the European credit card fraud data for this experiment. It is interesting to note that there appears to be diminishing returns as mtry grows large. One potential explanation may be that the overall ensemble of models becomes less diverse as each model is using similar sets of features to predict. Thus, keeping a reasonable degree of randomness in feature selection may carry important in performance implications.

We can observe the effect of increasing max.it on average model performance and average model training time in Figure 4. For this experiment, we trained 5 DeepBalance models with $m$ try $=5$ and total.nets $=5$ across max.it $=$ $20,30, \ldots, 100$. We then averaged model performance and train times. Overall, we see no gains in model performance, although we see a linear increase in train time. Thus, it may be beneficial for users to set max.it low. Early stopping with backpropagation has been shown to help neural networks generalize better [41]. 

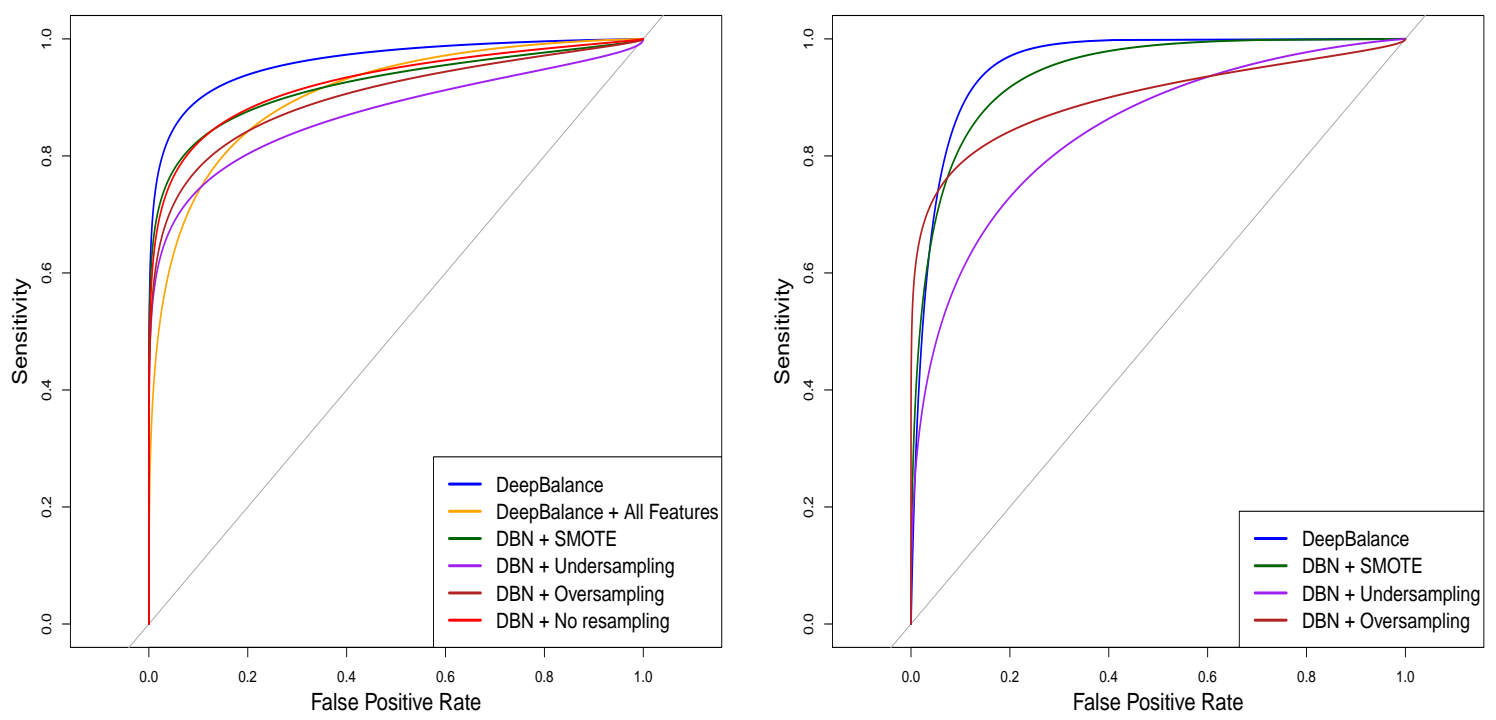

Fig. 3. Smoothed ROC Curves for European credit card fraud (left) and PaySim (right)
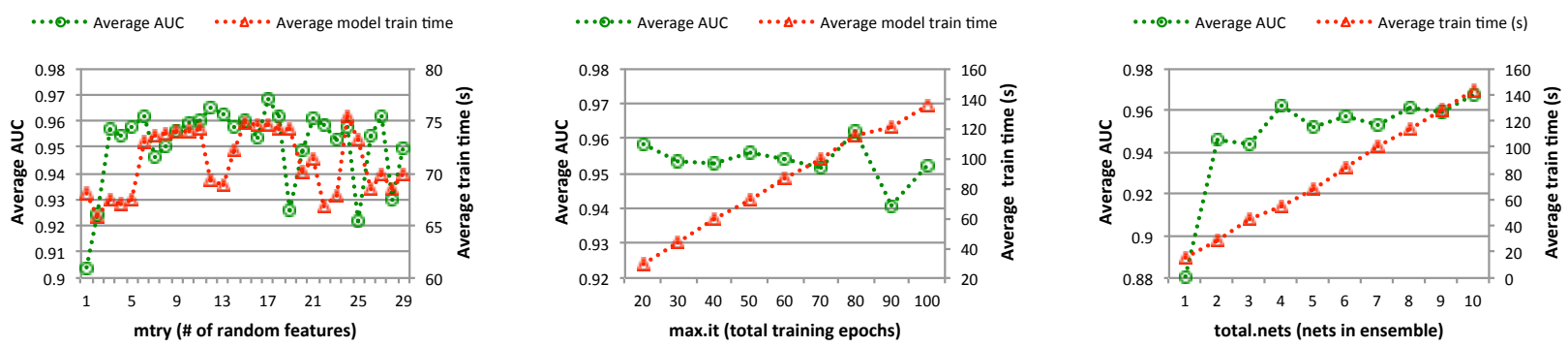

Fig. 4. Effects of changing mtry, max.it and total.nets on model performance (using European credit card fraud data)

We can assess the effect of increasing total.nets on average model performance and average model training time in Figure 5. For this experiment, we trained 5 DeepBalance models, each with mtry $=5$ and max.it $=50$ across total.nets of $1,2, \ldots, 10$. We see that in general, model performance increases as we add more DBNs to the ensemble. Such model improvement also comes at a training time hit, as it seems that complexity is linear in terms of total.nets. However, because training individual nets is an independent process, we can reduce the computational complexity coming from total.nets by introducing parallelism into the algorithm.

While computational complexity may increase through increasing mtry, total.nets or max.it, we can reduce the model training time through a parallel implementation of DeepBalance. Ensembling lends itself well to parallel implementation because each model in the ensemble is independent of the others. For example, each individual DBN can be trained on a separate processor. The trained models can then be sent back to the master process. If we keep the number of processors, $p$, equal to total.nets, then our complexity becomes solely dependent on mtry and max.it as we increase the number of networks we add to our ensemble. Future work will be directed at developing a parallel implementation of DeepBalance.

\section{CONCLUSION}

In this paper, we presented DeepBalance, an ensemble of deep belief networks trained with balanced bootstraps and random feature selection. Previous literature primarily focused on resampling methods for tree-based methods or costbased methods. We expanded existing literature by exploring the use of ensembles of deep belief networks in tackling imbalanced class problems in the absence of misclassification costs. We found that DeepBalance achieves slightly better performance than standard resampling methods in performance metrics such as sensitivity, balanced accuracy and AUC when applied to highly imbalanced financial transaction datasets. We believe this to result from the diverse ensemble that DeepBalance creates from both randomness in sampling and in feature selection.

Additionally, we explored the parameters of DeepBalance: mtry, max.it and total.nets. We see that total.nets has significant model performance implications, and as total.nets increases, model performance generally increases as ensemble diversity increases. We also observe that max.it 
and total.nets are linear in training times. The training time increase from training multiple models may be reduced by introducing parallelism into the training process.

Future direction will be directed towards exploring DeepBalance in a multiclass setting, benchmarking DeepBalance on various data sets and investigating other base learners. Ensemble methods have been shown to work well with resampling methods that balance out classes, and we will direct further efforts to a tool which allows the practitioner to select their base learner. Furthermore, we seek to investigate other variable selection techniques, by considering correlation among features in the sampling process or observing feature distributions conditional on the outcome class.

\section{REFERENCES}

[1] M. Kubat, R. C. Holte, and S. Matwin, "Machine learning for the detection of oil spills in satellite radar images," Machine Learning, vol. 30, no. 2-3, pp. 195-215, 1998.

[2] M. Kubat, R. Holte, and S. Matwin, "Learning when negative examples abound," Machine Learning: ECML-97, pp. 146-153, 1997.

[3] J. Burez and D. Van den Poel, "Handling class imbalance in customer churn prediction," Expert Systems with Applications, vol. 36, no. 3, pp. 4626-4636, 2009.

[4] S. Wang and X. Yao, "Using class imbalance learning for software defect prediction," IEEE Transactions on Reliability, vol. 62, no. 2, pp. 434-443, 2013.

[5] N. V. Chawla, "Data mining for imbalanced datasets: An overview," in Data Mining and Knowledge Discovery Handbook, pp. 875-886, Springer, 2009.

[6] R. Akbani, S. Kwek, and N. Japkowicz, "Applying support vector machines to imbalanced datasets," Machine Learning: ECML 2004, pp. 39-50, 2004.

[7] G. M. Weiss and F. Provost, "The effect of class distribution on classifier learning: an empirical study," Rutgers Univ, 2001.

[8] N. Japkowicz, "The class imbalance problem: Significance and strategies," in Proc. of the Intl Conf. on Artificial Intelligence, 2000.

[9] N. Japkowicz and S. Stephen, "The class imbalance problem: A systematic study," Intelligent Data Analysis, vol. 6, no. 5, pp. 429-449, 2002.

[10] H. He and E. A. Garcia, "Learning from imbalanced data," IEEE Transactions on Knowledge and Data eEngineering, vol. 21, no. 9, pp. 1263-1284, 2009.

[11] Z.-H. Zhou and X.-Y. Liu, "Training cost-sensitive neural networks with methods addressing the class imbalance problem," IEEE Transactions on Knowledge and Data Engineering, vol. 18, no. 1, pp. 63-77, 2006.

[12] C. Drummond, R. C. Holte, et al., "C4. 5, class imbalance, and cost sensitivity: why under-sampling beats over-sampling," in Workshop on Learning from Imbalanced Datasets II, vol. 11, Citeseer Washington DC, 2003.

[13] N. V. Chawla, K. W. Bowyer, L. O. Hall, and W. P. Kegelmeyer, "Smote: synthetic minority over-sampling technique," Journal of Artificial Intelligence Research, vol. 16, pp. 321-357, 2002.

[14] N. Lunardon, G. Menardi, N. Torelli, M. N. Lunardon, and M. Suggests, "Package rose," 2013.

[15] R. Mollineda, R. Alejo, and J. Sotoca, "The class imbalance problem in pattern classification and learning," in II Congreso Español de Informática (CEDI 2007). ISBN, pp. 978-84, 2007.

[16] G. E. Batista, R. C. Prati, and M. C. Monard, "A study of the behavior of several methods for balancing machine learning training data," $A C M$ Sigkdd Explorations Newsletter, vol. 6, no. 1, pp. 20-29, 2004.

[17] S. Kotsiantis, D. Kanellopoulos, P. Pintelas, et al., "Handling imbalanced datasets: A review," GESTS International Transactions on Computer Science and Engineering, vol. 30, no. 1, pp. 25-36, 2006.

[18] H. Han, W.-Y. Wang, and B.-H. Mao, "Borderline-smote: a new over-sampling method in imbalanced data sets learning," Advances in Intelligent Computing, pp. 878-887, 2005.

[19] N. Chawla, A. Lazarevic, L. Hall, and K. Bowyer, "Smoteboost: Improving prediction of the minority class in boosting," Knowledge Discovery in Databases: PKDD 2003, pp. 107-119, 2003.
[20] R. Batuwita and V. Palade, "Class imbalance learning methods for support vector machines," 2013.

[21] Y. Tang, Y.-Q. Zhang, N. V. Chawla, and S. Krasser, "Svms modeling for highly imbalanced classification," IEEE Transactions on Systems, Man, and Cybernetics, Part B (Cybernetics), vol. 39, no. 1, pp. 281288, 2009.

[22] X. Guo, Y. Yin, C. Dong, G. Yang, and G. Zhou, "On the class imbalance problem," in Fourth International Conference on Natural Computation, vol. 4, pp. 192-201, IEEE, 2008.

[23] J. Van Hulse, T. M. Khoshgoftaar, and A. Napolitano, "Experimental perspectives on learning from imbalanced data," in Proceedings of the 24th International Conference on Machine Learning, pp. 935-942, ACM, 2007.

[24] C. Chen, A. Liaw, and L. Breiman, "Using random forest to learn imbalanced data," University of California, Berkeley, vol. 110, 2004.

[25] X.-Y. Liu, J. Wu, and Z.-H. Zhou, "Exploratory undersampling for class-imbalance learning," IEEE Transactions on Systems, Man, and Cybernetics, Part B (Cybernetics), vol. 39, no. 2, pp. 539-550, 2009.

[26] P. Sollich and A. Krogh, "Learning with ensembles: How overfitting can be useful," in Advances in Neural Information Processing Systems, pp. 190-196, 1996.

[27] M. Galar, A. Fernandez, E. Barrenechea, H. Bustince, and F. Herrera, "A review on ensembles for the class imbalance problem: bagging, boosting-, and hybrid-based approaches," IEEE Transactions on Systems, Man, and Cybernetics, Part C (Applications and Reviews), vol. 42, no. 4, pp. 463-484, 2012.

[28] G. E. Hinton, S. Osindero, and Y.-W. Teh, "A fast learning algorithm for deep belief nets," Neural Computation, vol. 18, no. 7, pp. 1527$1554,2006$.

[29] G. E. Hinton and R. R. Salakhutdinov, "Reducing the dimensionality of data with neural networks," Science, vol. 313, no. 5786, pp. 504507, 2006.

[30] A.-r. Mohamed, G. Dahl, and G. Hinton, "Deep belief networks for phone recognition," in NIPS Workshop on Deep Learning for Speech Recognition and Related Applications, vol. 1, p. 39, Vancouver, Canada, 2009.

[31] A. Krizhevsky and G. Hinton, "Convolutional deep belief networks on cifar-10," Unpublished manuscript, vol. 40, 2010.

[32] R. Sarikaya, G. E. Hinton, and A. Deoras, "Application of deep belief networks for natural language understanding," IEEE/ACM Transactions on Audio, Speech and Language Processing (TASLP), vol. 22, no. 4, pp. 778-784, 2014.

[33] X. Qiu, L. Zhang, Y. Ren, P. N. Suganthan, and G. Amaratunga, "Ensemble deep learning for regression and time series forecasting," in Computational Intelligence in Ensemble Learning (CIEL), 2014 IEEE Symposium on, pp. 1-6, IEEE, 2014.

[34] C. Zhang, J. H. Sun, and K. C. Tan, "Deep belief networks ensemble with multi-objective optimization for failure diagnosis," in Systems, Man, and Cybernetics (SMC), 2015 IEEE International Conference on, pp. 32-37, IEEE, 2015.

[35] L. Yu, Z. Yang, and L. Tang, "A novel multistage deep belief network based extreme learning machine ensemble learning paradigm for credit risk assessment," Flexible Services and Manufacturing Journal, vol. 28, no. 4, pp. 576-592, 2016.

[36] A. Dal Pozzolo, O. Caelen, R. A. Johnson, and G. Bontempi, "Calibrating probability with undersampling for unbalanced classification," in Computational Intelligence, 2015 IEEE Symposium Series on, pp. 159-166, IEEE, 2015.

[37] E. Lopez-Rojas, A. Elmir, and S. Axelsson, "Paysim: A financial mobile money simulator for fraud detection," in 28th European Modeling and Simulation Symposium, EMSS, Larnaca, pp. 249-255, Dime University of Genoa, 2016.

[38] L. Torgo, Data Mining with R, learning with case studies. Chapman and Hall/CRC, 2010.

[39] J. Davis and M. Goadrich, "The relationship between precision-recall and roc curves," in Proceedings of the 23rd International Conference on Machine Learning, pp. 233-240, ACM, 2006.

[40] M. Drees, "Implementierung und analyse von tiefen architekturen in r," Master's thesis, Fachhochschule Dortmund, 2013.

[41] R. Caruana, S. Lawrence, and C. L. Giles, "Overfitting in neural nets: Backpropagation, conjugate gradient, and early stopping," in Advances in Neural Information Processing Systems, pp. 402-408, 2001. 